\title{
LÁSER DE DIODO; OPCIÓN TERAPÉUTICA PARA LIQUEN PLANO EROSIVO RESISTENTE A CORTICOTERAPIA: REPORTE DE CASO
}

\section{LASER DIODE; THERAPEUTIC OPTION FOR LICHEN PLANUS EROSIVE RESISTANT TO CORTICOTHERAPY: A CASE REPORT}

\author{
TÍTULO CORTO: LÁSER DE DIODO; OPCIÓN TERAPÉUTICA PARA LIQUEN PLANO \\ EROSIVO
}

Sandra Milena Espitia-Nieto ${ }^{1}$, Miguel Ángel Gómez-Romero², Javier Andrés Vargas-Pérez ${ }^{3}$

Recibido en marzo 24 de 2015

Aceptado en mayo 29 de 2015

\section{RESUMEN}

La terapia láser es una nueva opción de tratamiento por sus propiedades analgésicas, anti-inflamatorias y bioestimulantes y sin efectos adversos significativos, para patologías como el liquen plano oral en su forma erosiva, caracterizada por presentar úlceras crónicas y dolorosas con posibilidad de malignización, que obliga a una terapia adecuada. Este artículo reporta el caso clínico teniendo en cuenta las consideraciones éticas enmarcadas en la ley 008430 de 1993 de una paciente femenina de 50 años de edad con diagnóstico clínico e histopatológico de liquen plano oral erosivo que recibió tratamiento inicial con corticoides local y sistémico sin mejoría en siete meses; razón por la cual se optó por terapia de bioestimulación con láser de diodo 980nm, mostrando reducción significativa en la gravedad de las lesiones y disminución del dolor desde el primer ciclo de irradiación. El láser de diodo es un tratamiento seguro y efectivo en este tipo de enfermedades porque promueve la cicatrización, por tanto, puede ser considerado como una excelente alternativa en casos de liquen plano oral resistentes a la corticoterapia.

Palabras clave: liquen plano oral; láseres de diodo; efectividad; tratamiento

\section{ABSTRACT}

Laser therapy is a new treatment option for its analgesic, anti-inflammatory and bio-stimulants properties and without significant adverse effects, for diseases such as oral Lichen Planus in its erosive form, characterized by chronic and painful ulcers with possibility of malignization, which obliges an adequate therapy. This article reports the clinical case taking into account ethical considerations framed in law 008430 of 1993 of a female patient 50 years old, with clinical and histopathological diagnosis of oral erosive Lichen Planus, who received initial treatment with corticoids local and systemic without improvement in 7 months; reason why is opted biostimulation with 980nm

1. Estomatóloga y cirujana oral. Docente en la Universidad del Magdalena, Colombia. Correo: sandraepitia6@hotmail.com

2. Estudiante de último semestre de Odontología, Universidad del Magdalena, Colombia. Correo: migue1779@hotmail.com

3. Estudiante de último semestre de Odontología, Universidad del Magdalena, Colombia. Correo: javivperez27@gmail.com 
diode laser therapy, showing a significant reduction in the severity of injuries and decrease of pain from the first cycle of irradiation. Diode laser is safe and effective in this type of diseases treatment because it promotes healing, therefore it can be considered as an excellent alternative in cases of oral Lichen Planus resistant to corticotherapy.

Keywords: oral lichen llanus; diode lasers; effectiveness; treatment

\section{INTRODUCCIÓN}

$\mathrm{E}_{\mathrm{i}}^{\mathrm{l}}$ liquen plano oral (LPO) es una enfermedad inflamatoria mucocutánea crónica de etiología desconocida y relativamente común, con una prevalencia que varía de $0,5 \%$ a $2 \%^{1}$. Es más común en mujeres adultas $^{2}$. Se han descrito manifestaciones reticulares asintomáticas, atróficas y erosivas dolorosas ${ }^{3}$ con potencial maligno. La localización más frecuentemente es la lengua, pero puede aparecer en cualquiera de las mucosas orales ${ }^{4,5}$. El diagnóstico se basa en la evidencia clínica e histológica ${ }^{6}$. No hay ningún tratamiento definitivo para la LPO sintomático, los corticosteroides corresponden al tratamiento empírico más ampliamente aceptado, ya que alivian los síntomas ${ }^{7}$, disminuyen el dolor y permiten períodos de remisión, pero en lesiones crónicas el uso frecuente de estos fármacos origina efectos secundarios que afectan la morbilidad del paciente 0 pueden generar resistencia al tratamiento. Edwards y Kelsch $^{8}$ a través de un seguimiento promedio de 1,5 a 10 años, comprobaron que la relación de transformación maligna es de $0 \%$ a $10 \%$, con mayor riesgo en las formas atróficas y erosivas, posiblemente por la exposición de las capas más profundas del epitelio oral con agentes carcinógenos ${ }^{9}$. Los avances de la ciencia en el tratamiento de patologías orales, han proporcionado tecnologías innovadoras, como es el caso del láser de baja intensidad, que ha sido idóneo en el tratamiento de lesiones orales por sus ventajas bioestimulantes ${ }^{10}$, su uso en odontología se ha propuesto recientemente para el tratamiento del LPO ${ }^{11,12}$ como un tratamiento alternativo y eficaz reportado por autores como Cafaro et al. ${ }^{3}$ y Misra ${ }^{11}$ quienes demostraron mejoría significativas de la lesiones, sin complicaciones

La bioestimulación es un proceso inducido por la irradiación de baja intensidad, que se caracteriza por su capacidad para inducir efectos estimulantes ${ }^{10}$. El láser de diodo (DL), con longitud de onda entre 655 y 980 $\mathrm{nm}$, puede acelerar la cicatrización de heridas a través de la facilitación de la síntesis de colágeno, promoción de la angiogénesis y el aumento de la liberación del factor de crecimiento ${ }^{13}$. Los efectos biológicos de la radiación dependen de la longitud de onda, energía, tiempo de exposición y la irradiación dosis o fluencia. Pequeñas dosis inducen estímulos reparativos, grandes dosis deprime procesos fisiológicos, al extremo causan destrucción de estructuras biológicas ${ }^{10}$. El diodo es un semiconductor que utiliza elementos de estado sólido, tales como galio, arsenio, aluminio, y el indio ${ }^{14}$, para cambiar la energía eléctrica en energía luminosa. La energía de la luz del diodo se absorbe en gran medida por el tejido blando ${ }^{14}$.

\section{REPORTE DE CASO}

Se presenta el caso de una paciente femenina de 50 años de edad, quien consulta a estomatología con diagnóstico clínico e histológico de liquen plano oral erosivo de dos meses de evolución, como información relevante de sus antecedentes médicos-personales, refiere alergia a los aines y estrés emocional por algunos conflictos personales, niega enfermedades sistémicas, ingesta de fármacos y vicios. El examen físico intraoral evidencio mucosas de color rojo intenso, presencia de múltiples ulceraciones. Zonas erosivas-descamativas en encía anteros superiores e inferiores y atróficas en lengua, sintomáticos clasificada según escala visual análoga (EVA) en 9 con intolerancia a alimentos cítricos y especias (Figura 1 y 2). Refiere que previa terapia periodontal, iniciaron tratamiento con propionato de clobetasol tópico $(0,05 \%)$ en crema, mezclada con xilocaina en gel de 20mg, cápsulas de vitamina E de 400.000UI, sucralfato suspensión oral $1 \mathrm{gr}$ en $5 \mathrm{~mm}$ y nistatina $100.000 \mathrm{UI}$ en suspensión oral/ml, para evitar sobreinfección por cándida. Este tratamiento se usó por tres meses sin lograr mejoría y con aumento de estomatodinia y disminución de la ingesta de alimentos afectando su calidad de vida. Por tanto, se recomendó asistencia psicológica para manejo del estrés el cual fue rechazado por la paciente e interconsulta con dermatología, quien ordena dos ciclos de prednisolona tabletas de $5 \mathrm{mg}$ y corticoide tópico en 
orabase, reportando mejoría parcial de los síntomas, pero con recidiva inmediata postratamiento y molestias gastrointestinales. Dos meses después del tratamiento con corticoides sin buenos resultados, se opta por terapia láser pulsado con Diodo (GaAIAs) de 980nm, (distribuido por Giga láser).Siguiendo las instrucciones del fabricante y previa calibración. Utilizando pieza de mano de bioestimulación con un tamaño de punto de 3 $\mathrm{cm}^{2}$, densidad de potencia $1,5 \mathrm{~W} / \mathrm{cm}^{2}$ y una potencia de salida de $300 \mathrm{~mW}$.

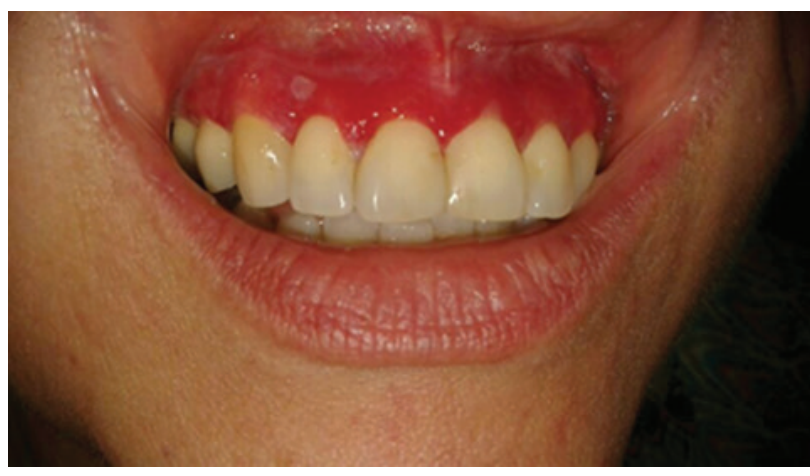

Figura 1. Se observa encía de coloración roja intensa y ulceración a nivel del órgano dental 12.

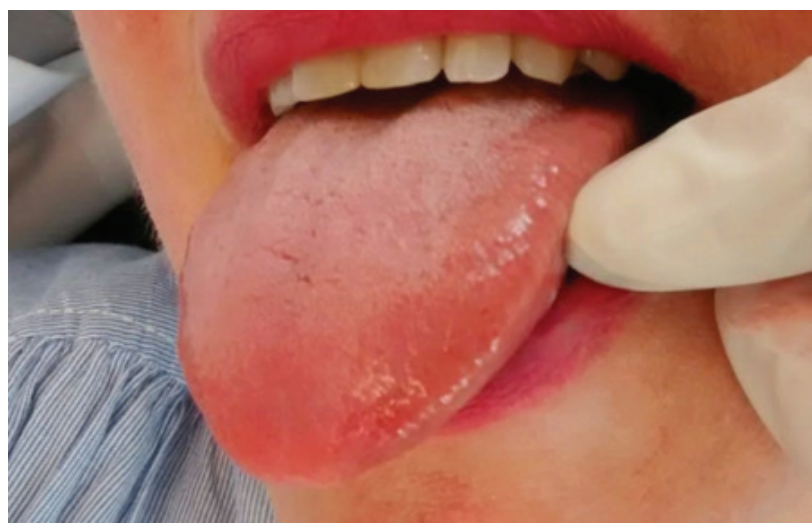

Figura 2. Se observa cara lateral izquierda de lengua con lesiones atróficas

Se realizó técnica irradiación puntual local en un área de $10 \mathrm{~cm}^{2}$ y colocando la pieza perpendicular a una distancia de $2 \mathrm{~mm}$ con movimiento de barrido hasta completar toda el área. Cada sesión fue realizada con una fluencia de $4,5 \mathrm{~J} / \mathrm{cm}^{2}$ por lesión. La aplicación de la irradiación se efectuó dos veces por semana por seis semanas con alivio del dolor desde la primera semana (EVA 1), cambio de coloración de las mucosas a rosa pálido. En la tercera semana de tratamiento, se observó disminución de lesiones erosivas y atróficas con permanencia de lesiones reticulares (Figura 3 y 4). Durante las siguientes aplicaciones se controló la evolución hasta la sexta semana donde se evidencio la resolución de lesiones erosivas- atróficas en encía y lengua, con predominio de lesiones reticulares para un total de 12 sesiones. Al mes de interrupción del tratamiento, el control mostró mucosas rosa pálida, asintomáticas, sin lesiones erosivas-atróficas y persistencia de algunas lesiones reticulares por lo que se recomienda cambio de obturaciones en amalgama, proceso en el que la paciente se encuentra al igual que controles mensuales.

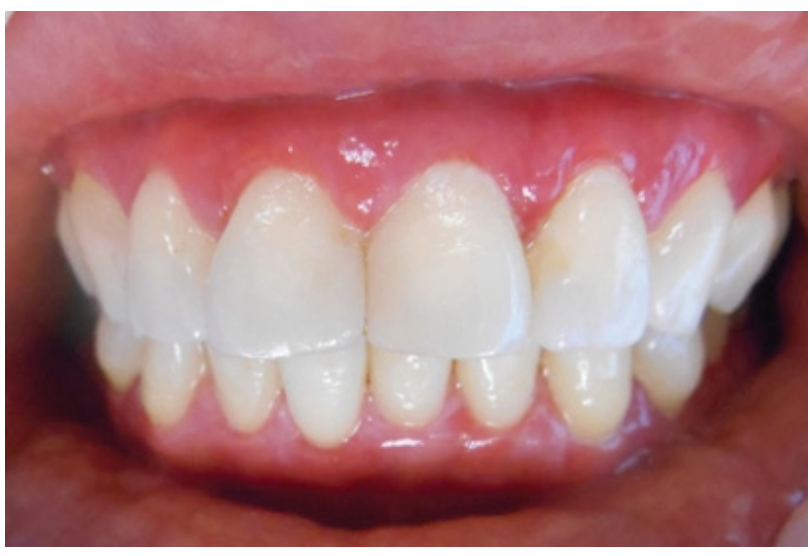

Figura 3. Se observa notoria mejoría después del tratamiento con láser de diodo en lesiones erosivas.

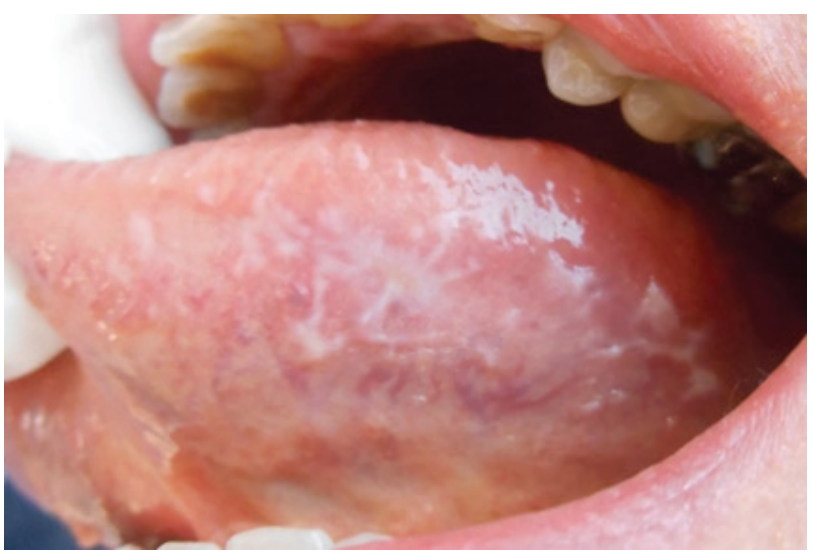

Figura 4. Se observan lesiones reticulares en lengua donde existía predominio de lesiones atróficas. 


\section{DISCUSIÓN}

El LPO tiene reporte de mayor prevalencia después del quinto decenio de la vida y las mujeres son más propensas a este tipo de enfermedad ${ }^{15}$, coincidiendo con el caso reportado. Los corticosteroides son la primera elección terapéutica para el LPO, sin embargo en la paciente no hubo mejoría de los síntomas ni en la evolución de la enfermedad, creando resistencia a estos, concordando con Cafaro et al. ${ }^{3}$, quienes trataron pacientes refractarios a los corticosteroides ${ }^{3}$, por lo que consideraron un punto fundamental en la elección del DL como terapia de primera opción ante el LPO.

La incursión del DL da una nueva perspectiva para obtener mejores resultados en cuanto a la mejoría de las lesiones si se compara con los corticosteroides como lo describe Dillenburg et $\mathrm{al}^{1}$, quienes muestran que el clobetasol a pesar de tener un buen comportamiento en el manejo de la lesión causa efectos secundarios y cuando es suspendido la patología rápidamente aparece, mientras que la terapia láser no muestra ningún efecto secundario y una vez terminada continua un patrón de mejora ${ }^{1}$. Autores como Bjordal et al. ${ }^{16} \mathrm{y}$ Peplow et al. ${ }^{17}$ han sugerido que los efectos positivos de DL podrían deberse a la disminución de las prostaglandinas E2, endoperóxido sintetasa 2 , interleukina 1 beta, factor de necrosis tumoral alfa, estrés oxidativo; así como la estimulación de la mitocondria para aumentar la producción de adenosin trifosfato incrementando las especies reactivas de oxígeno e incrementando la proliferación celular ${ }^{16,17}$.

La terapia de baja potencia con DL es una opción eficaz en el manejo del LPO erosivo en pacientes con fracasos con corticoides. Mostrando un resultado exitoso en la eliminación de lesiones erosivas con posibilidad de malignización ${ }^{18}$.

\section{Consideraciones éticas}

Teniendo en cuenta las consideraciones éticas enmarcadas en la ley 008430 de 1993, se le informó a la paciente de los procedimientos a realizar, materiales e instrumental que se va a utilizar, el objetivo de la investigación a publicar. Por lo cual, ella accede al tratamiento y a la publicación de las fotos y resultados obtenidos, firmando consentimiento informado.

\section{DECLARACIÓN SOBRE CONFLICTO DE INTERÉS}

Se deja constancia que los autores no tienen conflictos de interés y que este trabajo no ha tenido fuentes de financiamiento en ninguna de sus etapas.

\section{REFERENCIAS BIBLIOGRÁFICAS}

1. Dillenburg C, Martins M, Munerato M, Marques M, Carrard V, Sant'Ana M, et al. Efficacy of laser phototherapy in comparison to topical clobetasol for the treatment of oral lichen planus: a randomized controlled trial. Journal of Biomedical Optics. 2014; 19(6): 068002-068002.

2. Accurso B, Warner B, Knobloch T, Weghorst C, Shumway $\mathrm{B}$, Allen $\mathrm{C}$, et al. Allelic imbalance in oral lichen planus and assessment of its classification as a premalignant condition. Oral Surgery, Oral Medicine, Oral Pathology, Oral Radiology, and Endodontology. 2011; 112(3): 359-366.

3. Cafaro A, Arduino P, Massolini G, Romagnoli E, Broccoletti R. Clinical evaluation of the efficiency of lowlevel laser therapy for oral lichen planus: a prospective case series. Lasers in medical science. 2014; 29(1): 185-190.

4. Carbone M, Arduino P, Carrozzo M, Gandolfo S, Argiolas M, Bertolusso G, et al. Course of oral lichen planus: a retrospective study of 808 northern Italian patients. Oral diseases. 2009; 15(3): 235-243.

5. Lanfranchi-Tizeira H, Aguas S, Sano S. Malignant transformation of atypical oral lichen planus: a review of 32 cases. Medicina oral: organo oficial de la Sociedad Espanola de Medicina Oral y de la Academia Iberoamericana de Patologia y Medicina Bucal. 2002; 8(1) 2-9.

6. Sivolella S, Berengo M, Cernuschi S, Valente M. Diode laser treatment is effective for plaque-like lichen planus of the tongue: a case report. Lasers in Medical Science. 2012; 27 (2): 521-524.

7. Agha-Hosseini F, Moslemi E, Mirzaii-Dizgah I. Comparative evaluation of low-level laser and CO 2 laser in treatment of patients with oral lichen planus. International journal of oral and maxillofacial surgery. 2012; 41(10): 1265-1269.

8. Edwards P, Kelsch R. Oral lichen planus: clinical presentation and management. J Can Dent Assoc. 2002; 68 (8): 494-499. 
9. Jajarm H, Falaki F, Mahdavi O. A comparative pilot study of low intensity laser versus topical corticosteroids in the treatment of erosive-atrophic oral lichen planus. Photomed Laser Surg. 2011; 29(6): 421-425.

10. Santos H, Lamas R, Ciancaglini P. Solubilization of Na,K-ATPase from rabbit kidney outer medulla using only C12E8 Braz. J. Med. Biol. Res.2002; 35 (3): 277-288.

11. Misra N, Chittoria N, Umapathy D, Misra P. Efficacy of diode laser in the management of oral lichen planus. BMJ Case Rep.2013; (bcr): 2012007609.

12. Passeron T, Zakaria W, Ostovari N, Mantoux F, Lacour J, Ortonne J. Treatment of erosive oral lichen planus by the $308 \mathrm{~nm}$ excimer laser. Lasers in surgery and medicine. 2004; 34 (3): 205-205.

13. Sgolastra F, Severino M, Gatto R, Monaco A. Effectiveness of diode laser as adjunctive therapy to scaling root planning in the treatment of chronic periodontitis: a meta-analysis. Lasers Med Sci, 2013; 28 (5): 1393-1402.

14. Akbulut N, Kursun E, Tumer M, Kamburoglu K, Gulsen $\mathrm{U}$. Is the 810 -nm diode laser the best choice in oral soft tissue therapy? European Journal of Dentistry. 2013; 7 (2): 207.
15. Blanco A, Gándara J, Rodríguez A, García A, Rodríguez I. Alteraciones bioquímicas y su correlación clínica con el liquen plano oral. Medicina Oral. 2000; 5 (4): 238-249.

16. Bjordal J, Johnson M, Iversen V, Aimbire F, LopesMartins R. Low-level laser therapy in acute pain: a systematic review of possible mechanisms of action and clinical effects in randomized placebo-controlled trials. Photomed Laser Surg. 2006; 24 (2):158-168.

17. Peplow P, Chung T, Baxter G. Laser photobiomodulation of wound healing: a review of experimental studies in mouse and rat animal models. Photomed laser surg. 2010; 28 (3): 291-325.

18. Hsue S, Wang W, Chen C, Lin C, Chen Y, Lin L. Malignant transformation in 1458 patients with potentially malignant oral mucosal disorders: a follow-up study based in a Taiwanese hospital. Journal of oral pathology \& medicine. 2007; 36 (1): 25-29.

Para citar este artículo: Espitia-Nieto S, Gómez-Romero M, Vargas-Pérez J. Láser de diodo; opción terapéutica para liquen plano erosivo resistente a corticoterapia: reporte de caso. Duazary. 2016 enero; 13 (1): 47 - 51 